\title{
THE CONTRIBUTION OF BINARIES TO THE OBSERVED GALACTIC MICROLENSING EVENTS
}

\author{
M. DOMINIK AND A. C. HIRSHFELD \\ Institut für Physik, Universität Dortmund \\ D-44221 Dortmund, Germany
}

\begin{abstract}
Most of the detected galactic microlensing events are commonly explained as due to microlensing of a pointlike source by a pointlike lens. We have found statistical methods to determine the goodness-of-fit for a specific model of source and lens beyond the assumption that the errors are normal, which does not always hold. In particular, we argue that at least one weak binary lensing event has already been detected (MACHO LMC\#1) and thereby confirm our former hypothesis (Dominik \& Hirshfeld 1994). We also find that this fit is not unique. We emphasize that knowledge of the fraction of binaries among the observed microlensing events is crucial for estimating the mass distribution of the observed dark objects.
\end{abstract}

\section{Introduction}

To draw the correct conclusions from the observed galactic microlensing events it is important to know if the model of source and lens used suffices to describe the data. An insufficient model may lead to errors concerning the time- and mass scales. A standard $\chi^{2}$-test does not work when the photometry errors do not follow a normal distribution.

Our analysis therefore makes use of the following ideas to decide if a fit is reasonable or not

- Since the light curve should have a constant tail, we use the errors of the data points there to determine a constant rescaling factor.

- We allow distributions with larger tails having the form of Student's $t$-distribution.

- We look at the correlation between the deviations from the best-fit light curve of the data in two spectral bands. 


\section{Results}

We find for the MACHO LMC \#1 event that

- a goodness-of-fit analysis rejects fits for a pointlike lens with a point source and also with an extended circular source with uniform brightness

- the analysis of the correlation coefficient shows that a binary source is also unlikely to explain the event

- at least 6 binary lens models can be accommodated

We also find that

- OGLE \#7 is clearly a strong lensing event.

- We do not see a clear indication for a binary lens in the OGLE \#6 event; in particular we have found a binary source fit with a slightly lower $\chi^{2}$ than that quoted by Mao \& DiStefano (1995), which only shows an asymmetry, but no second peak.

- If we apply our rescaling procedure to the OGLE events assuming a normal distribution, we find that the OGLE \#4 and OGLE \#5 events are not acceptable as being due to microlensing of a point source by a pointlike lens.

- The EROS \#1 event is compatible with microlensing of a point source by a pointlike lens.

We think that it should in principle be possible for all of the events to learn about the distribution of the errors from the tails of the light curves in order to enable goodness-of-fit tests between different models of the peak.

The test of the correlation coefficient seems to be another promising tool to reject fits, but its use is limited to the events observed by the MACHO collaboration up to now, since observation in two spectral bands at the same time is necessary.

\section{Acknowledgements}

We would like to thank S. Mao for some interesting and fruitful discussions, the MACHO collaboration for making available the data of the MACHO LMC \#1 event over the computer network, the OGLE collaboration for making available the data of the OGLE \#1...\#7 events, the EROS collaboration, esp. A. Milsztajn, for sending us the data of the EROS \#1 event, A. Udalski for his help in retrieving a paper and P. Schneider for some advice.

\section{References}

Dominik, M., \& Hirshfeld, A. C. 1994, A\&A, 289, L31

Mao, S., \& Di Stefano, R. 1995, ApJ, 440, 22 